(C) 2007 The Japan Society of Applied Physics

\title{
Significantly Enhanced Birefringence of Photonic Crystal Fiber Using Rotational Binary Unit Cell in Fiber Cladding
}

\author{
Yuan-Fong ChaU*, Han-Hsuan YeH, and Din Ping TsaI ${ }^{1}$ \\ Department of Electronic Engineering, Ching Yun University, Jung Li, Taiwan 320, R.O.C. \\ ${ }^{1}$ Department of Physics and Center for Nanostorage Research, National Taiwan University, Taipei, Taiwan 106, R.O.C.
}

(Received July 11, 2007; accepted September 27, 2007; published online November 2, 2007)

We propose a novel high-birefringence index-guiding photonic crystal fiber (PCF). This PCF is composed of a solid silica core and a cladding with rotational squeezed-triangular-lattice elliptical air holes, which consist of binary unit cells. The birefringence of a fundamental mode in such a PCF is analyzed numerically using the finite element method. A binary unit cell in a PCF cladding that combines its rotational effects can enhance the birefringence as high as a magnitude of the order of $10^{-2}$. This study provides a new viewpoint for the characterization and design of a high-birefringence PCF.

[DOI: 10.1143 /JJAP.46.L1048]

KEYWORDS: photonic crystal fiber (PCF), high birefringence, binary unit cell, finite element method

In recent years, photonic crystal fibers (PCFs) have attracted considerable interest as an alternative to conventional optical fibers in manipulating the properties of light and the applications in optical devices. ${ }^{1-6)}$ Due to the design flexibility and high index contrast, highly birefringent (Hi-Bi) PCFs can easily be realized. Many research studies on PCFs with the enhancement of their birefringence. One possible use of $\mathrm{Hi}-\mathrm{Bi} \mathrm{PCF}$ is as polarization-maintaining fibers (PMFs), which can eliminate the effect of polarization mode dispersion or stabilize the operation of optical devices.

However, most of the current PMFs typically show a modal birefringence of the order of $10^{-4}$. In recent years, different approaches for achieving high birefringence have been explored for PCFs. Hi-Bi index-guiding PCFs with holes of different diameters along the two orthogonal fiber axes or with asymmetric core designs have been proposed. ${ }^{7-10)}$ Very recently, a tremendously $\mathrm{Hi}-\mathrm{Bi}$ polarizationmaintaining PCF has been proposed, ${ }^{6}$ and their numerical results showed a group birefringence of $3.78 \times 10^{-2}$. Due to the gaps between the holes being very narrow, the corresponding fiber structure with its optimal structural parameters is weak under external forces. This implies that a single unit cell (the same size as an air hole) in the PCF cladding has the drawback of a narrower gap between air holes, whose sections superimpose, making the structure physically unfeasible.

Motivated by these previous works cited here and inspired by these active issues, in this study, we propose a novel structure of a high-birefringence index-guiding PCF, which is based on our previous work. ${ }^{11)}$ This PCF is composed of a solid silica core and a cladding with rotational squeezedtriangular-lattice elliptical air holes. The proposed structure is used as a binary unit cell (two rotational elliptical air holes of different sizes of) to replace the single unit cell (one size of an elliptical air hole) in the PCF cladding. The binary unit cell of rotational elliptical air holes could provide a new degree of freedom to design birefringence in optical waveguides. In addition, owing to the gaps between the two different air holes (binary unit cell) being larger than that of single unit cell (e.g., the structure shown in ref. 6), the corresponding fiber structure designed using the binary unit cell in the PCF cladding is stronger than that of designed

*E-mail address: yfc01@cyu.edu.tw using the single unit cell under external forces. The birefringence of our proposed structure contributes to the entire cladding asymmetry. The fiber core is a point defect, which is formed by the omission of one small elliptical air hole of the center of this structure, whose mode field in the core region is well confined by the rotational effect of elliptical air holes in the PCF cladding; thus, it is possible to create a Hi-Bi PCF.

PCF optical fibers with elliptical holes were experimentally realized in 2004. ${ }^{12}$ ) With the development of new methods for fabricating PCFs, such as perfor drilling, sol-gel casting, and tapering, ${ }^{12,13)}$ it is possible for us to draw PCFs with such a structure. In this paper, we discuss the origin of the birefringence and analyze its dependence on structural parameters in the proposed PCFs with elliptical air hole of different sizes in a PCF cladding. We also discuss the effect of the rings of air holes on birefringence. To our knowledge, this is the first demonstration of a high-birefringence PCF using the binary unit cell of rotational elliptical air holes in a triangular PCF cladding. Among the full vectorial methods used in modeling PCFs, ${ }^{14-18)}$ the finite-element method (FEM) is particularly effect at handling curved interfaces with high accuracy and obviously is the good choice for combined elliptical shape and waveguide analysis. The numerical method used in this study is FEM, which is adequate for the analysis of general dielectric waveguide geometries. It has already been successfully applied to the investigation of the dispersion properties of triangular and cobweb PCFs. ${ }^{19,20)}$ The fiber cross sectional representation is very accurate as the domain is divided into triangular or quadrilateral subdomains, where any refractive index profiles can be properly represented. Calculations have been performed using consisteness FEM, as stated in refs. 21-24 in which this method is described in detail and its convergence and suitability for computing birefringence in arbitrarily shaped scatterers are also assessed. To modelize on infinite PCF with a two-dimensional finite-geometry model (i.e., to enclose the computational domain without affecting the numerical solution), it is necessary to use anisotropic perfectly matched layers (PMLs), which are placed before the outer boundary.

The cross section of the proposed PCF is shown in Fig. 1. We use the PCF with rotational elliptical air holes as the binary (complex) unit cell cladding; each lattice point forms 


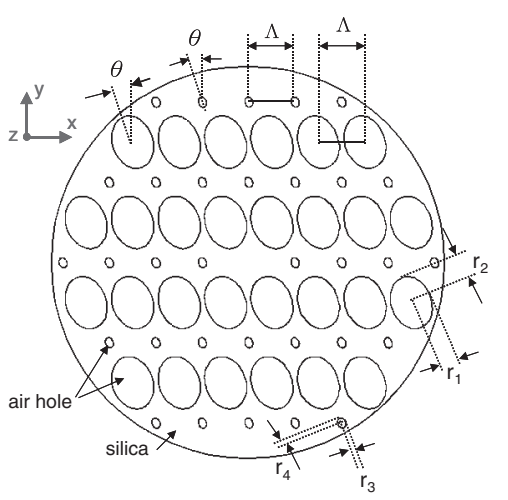

Fig. 1. Cross section of our proposed birefringent PCF.

the cladding consisting of a binary unit cell (two rotational elliptical air holes of different sizes) with a pitch (center to center distance between the holes), $\Lambda=1.96 \mu \mathrm{m}$. As shown in Fig. 1, $r_{1}$ and $r_{2}$ denote the half lengths of large elliptical holes along the $x$ - (minor axes) and $y$ - (major axis) directions, respectively. In the same manner, $r_{3}$ and $r_{4}$ represent the half lengths of small elliptical holes. The elliptical air holes are rotated by an angle of $\theta$. The core is formed by the omission of one small elliptical air hole at the center of such a structure. We also define the coefficient $\eta=r_{1} / r_{3}$ to determine the relationship between the large and small elliptical air holes. The refractive index of background silica is set to be $n=1.45$ and that of the air holes is set to be 1 in our simulation. In Fig. 1, birefringence is introduced by the difference between the two orthogonal directions. In the $x$ - and $y$-directions of two different linear, homogeneous, and isotropic dielectrics, Maxwell's equations impose boundary conditions for the normal and transverse components of magnetic fields. There is one important fact, that is, the mode fields in PCFs appear like the $H E_{11}$ mode in step-index fibers, which we refer to as $H E_{11 x}$ and $H E_{11 y}$. The doublet components of the fundamental mode, as we know, are degenerate in conventional standard and circular air-hole triangular lattice PCF fibers. However, when the air holes are elliptical, degeneracy splits significantly. Regardless of whether both modes are confined or leaky, birefringence can be defined as the refractive indices of the $x$ - and $y$-polarization modes, respectively.

To illustrate the field profile of our proposed PCFs, the fundamental mode of our designed fiber with various parameters, such as $\Lambda=1.96 \mu \mathrm{m}, r_{1}=0.9 \mu \mathrm{m}, r_{2}=1.2 \mu \mathrm{m}$, $r_{3}=0.164 \mu \mathrm{m}, \quad r_{4}=0.218 \mu \mathrm{m}, \quad \eta\left(r_{1} / r_{3}\right)=5.5$, elliptical ratio $r_{1} / r_{2}=r_{3} / r_{4}=0.75$, and rotational angle $\theta$ at the excitation wavelength $\lambda=1550 \mathrm{~nm}$, are shown in Fig. 2, illustrating the confinement of light in the PCFs. It can be observed in Fig. 2 that the $x$-polarized mode is stronger than the $y$-polarized model owing to the fact that $x$-polarized states have lower air filling fractions than $y$-polarized states. Obviously, the asymmetry formed by a binary unit cell cladding in PCFs is one of the key factors in determining the localization extent of the transverse mode. The simulation results for the $x$ - and $y$-polarized modes are strongly bounded in the high-index core region, giving the birefringence $\Delta n=\left|n_{\text {eff }}^{x}-n_{\text {eff }}^{y}\right|=|1.375547-1.366716|=8.831 \times 10^{-3}$ for the nonrotation binary unit cell [the case of $\theta=0^{\circ}$, see Fig. 2(a)] and the birefringence $\Delta n=\left|n_{\text {eff }}^{x}-n_{\text {eff }}^{y}\right|=$

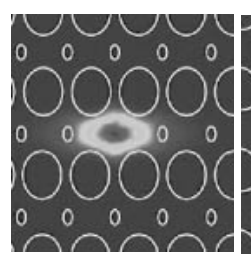

(a)

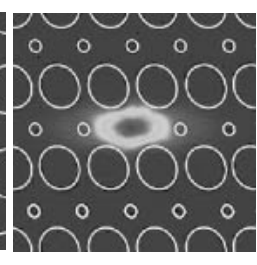

(b)
Fig. 2. Mode field pattern with various parameters, such as $\Lambda=1.96 \mu \mathrm{m}$, $r_{1}=0.9 \mu \mathrm{m}, r_{2}=1.2 \mu \mathrm{m}, r_{3}=0.164 \mu \mathrm{m}, r_{4}=0.218 \mu \mathrm{m}, \eta\left(r_{1} / r_{3}\right)=5.5$, elliptical ratio $r_{1} / r_{2}=r_{3} / r_{4}=0.75$ and rotated by angles of (a) $\theta=0$. and (b) $\theta=25^{\circ}$ at excitation wavelength $\lambda=1550 \mathrm{~nm}$.

$|1.380413-1.370249|=1.0164 \times 10^{-2}$ for the rotational binary unit cell angle of $\theta=25^{\circ}$ [see Fig. 2(b)], which is much higher than those obtained from a conventional stepindex fiber $\left(10^{-4}\right),{ }^{7}$ circular air holes $\left(10^{-3}\right),{ }^{3)}$ and elliptical hollow PCFs $\left(10^{-3}\right) .^{25)}$ Simulation results show that the rotational effect of the binary unit cell in the PCF cladding can significantly enhance birefringence. Another significant result from Fig. 2 is that the asymmetric core shape affects the polarization mode in PCFs and splits the fields extended far beyond the core-cladding interface. It is noteworthy to confirm that if a large birefringence is desired, the parameters of PCFs are limited by the requirement for mode profiles exhibiting high field intensities in the core region.

The effective index and birefringence of PCFs are relative to the varying wavelength. As expected, the difference between the two polarized direction modes in the cladding asymmetry causes a high birefringence in PCFs. Figure 3 shows birefringence as a function of wavelength ranging from $\lambda=0.8$ to $2.0 \mu \mathrm{m}$, whereas the hole spacing remains constant at $\Lambda=1.96 \mu \mathrm{m}, \eta=r_{1} / r_{3}=5.5$, and $r_{1} / r_{2}=$ $r_{3} / r_{4}=0.75$. It can be clearly observed that the birefringence is sensitive to the varying wavelength $\lambda$, and the birefringence of the rotational binary unit cell is higher than that of the nonrotational binary unit cell. As shown in Fig. 3, the family curves of birefringence shift upward, corresponding to an increase in wavelength. Namely, wavelength is also one of the key factors in determining the localization extent of the transverse mode. The dispersion curve of $\theta=5^{\circ}$ is very near the result of $\theta=0^{\circ}$, and the results of $\theta$ more than $15^{\circ}$ start to shift upward compared with that of $\theta=0^{\circ}$. The corresponding wavelength $\lambda=1.55 \mu \mathrm{m}$ with birefringence reaches its

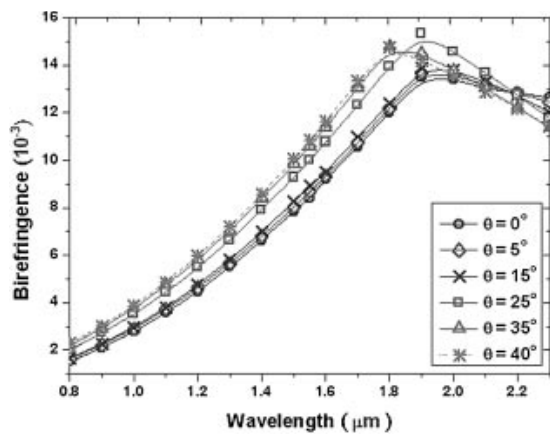

Fig. 3. Family curves of birefringence as function of wavelength ranging from $\lambda=0.8$ to $2.0 \mu \mathrm{m}$ with various rotational angles of binary unit cell, whereas structural parameters $\Lambda=1.96 \mu \mathrm{m}, \eta=r_{1} / r_{3}=4.5$, and $r_{1} / r_{2}=r_{3} / r_{4}=0.75$. 


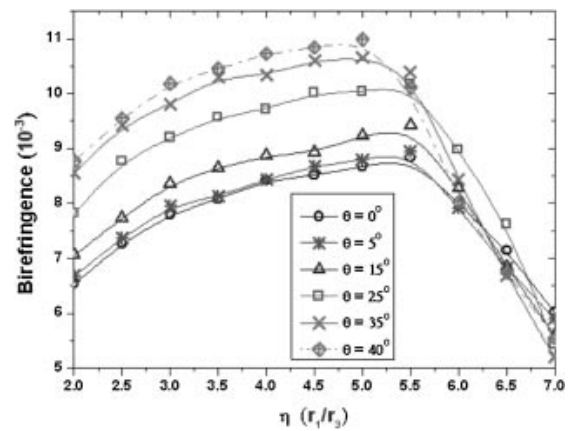

Fig. 4. Birefringence as function of minor axis ratio $\eta\left(r_{1} / r_{3}\right)$ of elliptical holes ranging from $\eta=2.0$ to $7.0 \mu \mathrm{m}$ at different rotational angles of the binary unit cell with structural parameters $\Lambda=1.96 \mu \mathrm{m}$ and the excitation wavelength $\lambda=1.55 \mu \mathrm{m}$.

maximum values $\Delta n=1.0837 \times 10^{-2}$ at $\theta=40^{\circ}$ and $\Delta n=$ $8.504 \times 10^{-3}$ at $\theta=0^{\circ}$, giving an enhancement of $0.2333 \times$ $10^{-3}$ due to the effect of the rotational binary unit cell. It can be indicated that rotating the angle of binary unit cell in the PCF cladding increases birefringence. The increment is much more significant at long wavelengths than at short wavelengths. With the appropriate rotational angle of the binary unit cell ranging from $\theta=15$ to $40^{\circ}$ for the example shown in Figs. 2 and 3, a high birefringence may be achieved.

We further studied the effect of elliptical air-hole angle on rotation-induced birefringence of PCFs. Figure 4 shows birefringence as a function of the minor axis ratio $\eta\left(r_{1} / r_{3}\right)$ of elliptical holes ranging from $\eta=2.0$ to 7.0 at different rotational angles of the binary unit cell with the structural parameters $\Lambda=1.96 \mu \mathrm{m}$ and $\lambda=1.55 \mu \mathrm{m}$. The results in Fig. 4 clearly show that the size variations of $\eta\left(r_{1} / r_{3}\right)$ may yield different birefringences at different rotational angles of the binary unit cell. The rotation-induced birefringence increases as the rotational angle of the binary unit cell increases in the $\eta\left(r_{1} / r_{3}\right)$ range of 2.0 to 5.0. When the size of $\eta\left(r_{1} / r_{3}\right)$ is larger than 5.5 , birefringence is markedly reduced. The corresponding wavelength $\lambda=1.55 \mu \mathrm{m}$ at $\eta\left(r_{1} / r_{3}\right)=5.0$, with birefringence reaches its maximum value $\Delta n=1.0986 \times 10^{-2}$ at $\theta=40^{\circ}$, and $\Delta n=8.671 \times$ $10^{-3}$ at $\theta=0^{\circ}$, giving an enhancement of $0.2315 \times 10^{-3}$ due to the effect of the rotational binary unit cell.

The effects of field confinement and its stability on birefrigence play a fundamental role in the optical properties of PCFs. They depend on air hole diameter, their pitch and the number of rings. In practice, more than 5 rings of air holes are often required to reduce the confinement loss to an acceptable level. For the accuracy, we use 10 rings in this paper. In the following analysis, we fix the wavelength $\lambda=1.55 \mu \mathrm{m}, \Lambda=1.96 \mu \mathrm{m}, r_{1} / r_{2}=r_{3} / r_{4}=0.75$, and the ratio of large and small air holes $\eta=r_{1} / r_{3}=4.5$. The rotational angles $\theta$ are varied from 0 to $40^{\circ}$. The effect of rotating the angle of the binary unit cell in the PCF cladding on birefringence stability is also significant, as shown in Fig. 5. It can clearly be observed that the higher the rotational angle $\theta$, the higher the birefringence. The birefringence of the rotational binary unit cell of elliptical air holes in PCF that is higher than that of the nonrotational binary unit cell, can be assumed to be due to the contribution of the rotation effect, which lead to more fields confined in the core region; thus, birefringence is more stable even in the case of 10 rings.

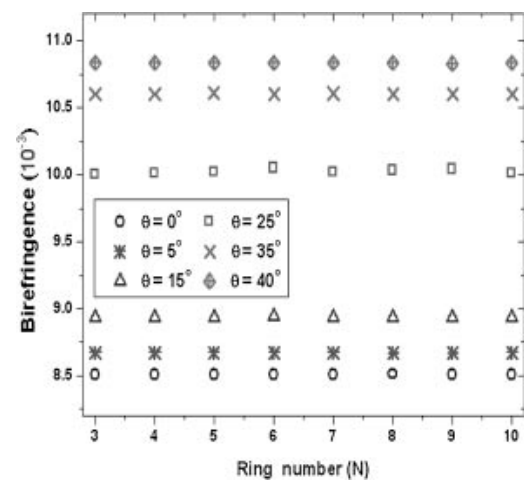

Fig. 5. Birefringence as function of number of rings at different rotational angles of binary unit cell cladding.

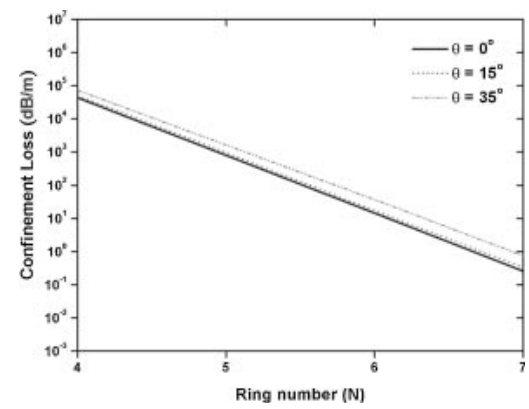

Fig. 6. Confinement loss as function of number of rings $(N=4-7)$.

FEM with PMLs, which are placed before the outer boundary can be used to calculate the confinement loss of PCFs. The imaginary part of complex effective index represents the loss. As described in refs. 25 and 26 for small holes and few hole rings, the confinement loss is huge, but decreases rapidly as air hole diameter increases or more hole rings are employed. Figure 6 shows the confinement loss with the fixed parameters, namely, $\lambda=1.55 \mu \mathrm{m}, \Lambda=$ $1.96 \mu \mathrm{m}, r_{1} / r_{2}=r_{3} / r_{4}=0.75$, and the number of rings ranging from $N=4$ to 7 (because $N=7$, the caculation of the confinement loss using FEM with PMLs is out of our computer memory). It can be observed in Fig. 6, that the confinement losses of at different rotational angles are $0.2557 \mathrm{~dB} / \mathrm{km}$ (for $\theta=0^{\circ}$ ), $0.3301 \mathrm{~dB} / \mathrm{km}$ (for $\theta=15^{\circ}$ ), and $0.7495 \mathrm{~dB} / \mathrm{km}$ (for $\theta=35^{\circ}$ ), when the number of air hole rings $N$ is 7 . This shows that the effect of rotational binary unit cell on the optical loss (mode confinement) is small, even in the case of rotational angle $\theta=35^{\circ}$.

In conclusion, a Hi-Bi index-guiding elliptical air-hole PCF with a rotational binary unit cell in a PCF cladding is successfully demonstrated numerically using the finite element method. The Hi-Bi PCF is assigned to the different sizes of elliptical air holes and the rotational effect. The rotational elliptical air holes as a binary unit cell in a PCF cladding could provide a new degree of freedom to design birefringence in optical waveguides. The birefringence of a fundamental mode in such a PCF is analyzed by utilizing the intrinsically large index contrast of the PCF in combination with rotational binary unit cell cladding designs. We have found that it is possible to enhance birefringence as high as a magnitude of the order of $10^{-2}$, which is at least two orders of magnitude higher than that for conventional Hi-Bi 
fibers ${ }^{7)}$ and one order of magnitude higher than that reported for other PCF structures. ${ }^{3,7,25)}$ There are many methods for inducing birefringence in PCFs. The key point is to destroy the symmetry of the structure and determine the effective index difference between the two orthogonal polarization states. The suggested structure possesses a high birefringence to separate the two polarization modes. Our simulation results provide valuable insight into the realization of a PCF with a higher birefringence than that indicated in the literature and give a new viewpoint for the characterization and design of high-birefringence PCFs.

The authors are thankful for the financial support from the National Science Council, Taiwan, R.O.C., under grant number NSC 96-2112-M-231-001-MY3 and NSC 96-2120M-002-017.

1) J. C. Knight, T. A. Birks, P. St. J. Russell, and D. M. Atkin: Opt. Lett. 21 (1996) 1547

2) T. A. Birks, J. C. Knight, and P. St. J. Russell: Opt. Lett. 22 (1997) 961.

3) A. Ortigosa-Blanch, J. C. Knight, W. J. Wadsworth, J. Arriaga, B. J. Mangan, T. A. Birks, and P. St. J. Russell: Opt. Lett. 25 (2000) 1325.

4) J. K. Ranka, R. S. Windeler, and A. J. Stentz: Opt. Lett. 25 (2000) 25.

5) Z. Zhu and T. G. Brown: Opt. Lett. 28 (2003) 2306.

6) Y. Yue, G. Kai, Z. Wang, T. Sun, L. Jin, Y. Lu, C. Zhang, J. Liu, Y. Li, Y. Liu, S. Yuan, and X. Dong: Opt. Lett. 32 (2007) 469.

7) J. Noda, K. Okamoto, and Y. Sasaki: J. Lightwave Technol. 4 (1986) 1071.

8) M. J. Steel and R. M. Osgood: J. Lightwave Technol. 19 (2001) 495.
9) K. Saitoh and M. Koshiba: IEEE Photonics Technol. Lett. 14 (2002) 1291.

10) A. Hochman and Y. Leviatan: Opt. Express 13 (2005) 6193.

11) Y.-S. Sun, Y.-F. Chau, H.-H. Yeh, T.-J. Yang, and D. P. Tsai: Appl. Opt. 46 (2007) 5276.

12) N. A. Issa, M. A. van Eijkelenborg, M. Fellew, F. Cox, G. Henry, and M. C. J. Large: Opt. Lett. 29 (2004) 1336.

13) P. Domachuk, A. Chapman, E. Magi, M. J. Steel, H. C. Nguyen, and B. J. Eggleton: Appl. Opt. 44 (2005) 3885.

14) A. Ferrando, E. Silvestre, J. Miret, and P. Andres: Opt. Lett. 24 (1999) 276.

15) D. Mogilevtsev, T. A. Birks, and P. St. J. Russell: J. Lightwave Technol. 17 (1999) 2078.

16) Z. Zhu and T. G. Brown: Opt. Commun. 206 (2002) 333.

17) Z. Zhu and T. Brown: Opt. Express 10 (2002) 853.

18) F. Brechet, J. Marcou, D. Pagnoux, and P. Roy: Opt. Fiber Technol. 6 (2000) 181.

19) A. Cucinotta, S. Selleri, L. Vincetti, and M. Zoboli: J. Lightwave Technol. 20 (2002) 1433.

20) A. Cucinotta, S. Selleri, L. Vincetti, and M. Zoboli: IEEE Photonics Technol. Lett. 14 (2002) 1530.

21) S. Selleri, L. Vincetti, A. Cucinotta, and M. Zoboli: Opt. Quantum Electron. 33 (2001) 359.

22) D. Ferrarini, L. Vincetti, M. Zoboli, A. Cucinotta, and S. Selleri: Opt. Express 10 (2002) 1314.

23) J. Jin: The Finite Element Method in Electromagnetics (John Wiley \& Sons, New York, 2002).

24) http://www.comsol.com/

25) Y. Jung, S. R. Han, S. Kim, U. C. Paek, and K. Oh: Opt. Lett. 31 (2006) 2681.

26) T. P. White, R. C. McPhedran, C. M. de Sterke, L. C. Botten, and M. J. Steel: Opt. Lett. 26 (2001) 1660.

27) A. Bétourné, V. Pureur, G. Bouwmans, Y. Quiquempois, L. Bigot, M. Perrin, and M. Douay: Opt. Express 15 (2007) 316. 